\title{
Asymmetric Continuum Theories - Fracture Processes in Seismology and Extreme Fluid Dynamics
}

\author{
Teisseyre Roman \\ Institute of Geophysics, Polish Academy of Sciences, Warszawa,
} Poland

\section{Introduction}

Our consideration has its origin in a well known fact that the classical continuum mechanics cannot well describe the seismic processes, seismic wave emission and even wave propagation. Classical elasticity describes perfectly the small deformations, but in its frame we cannot imagine the recording of the very long seismic displacement waves. In fact we record the deformation waves which, in case of a constant pressure load, directly relate to shear and rotation waves. Only in a more general theories, like the very complicated micromorphic theory, the rotation waves may exist: some recent observation data, obtained by very sensitive sensors, confirm existence of the rotational waves. Our aim is to present a relatively simple theoretical frame, based on the Newton law (exactly its first order derivatives) applied for the deformation strains (first order derivatives of displacements); in this way we arrive directly to the fundament of the Asymmetric Continuum Theory. Following this way we like also to present an approach to Asymmetric Molecular Strain Theory, such way may lead us to some explanations related to the vortex problems in fluids and even mechanism of turbulence.

The Asymmetric Continuum Theory (Teisseyre, 2008, 2009, 2011) has been developed due to many insufficiencies of the classic symmetric theory and some phenomena found by seismological observations, e.g., the trials to record the rotation motions has started already at beginning of last century, while only at end of that century such reliable records have been discovered by means of the Sagnac measurement system (cf., Lee et al., 2009).

The similar asymmetric approach can be introduced for the fluids; the molecular strains may present the symmetric and anti-symmetric molecular deformations (Teisseyre, 2009). However, we will meet there the additional complications when considering the nonlaminar and turbulent motions; moreover, the density variation in gases will have a great importance. We will try to present here some related trials.

However, firstly we may shortly explain the difficulties found with an use of the classic elasticity:

- Classic elasticity include only displacements as basic motions; rotations and strains do not appear as independent deformations. The independently released rotation and 
strain fields should have the own independent motion relations, e.g., a balance of angular momentum shall be independently introduced, while in the classical elasticity this balance holds automatically due to the symmetry of stresses;

- Fault slip solutions used in seismology could be included only by the additional friction constitutive laws;

- Fracture pattern reveals usually an asymmetric pattern with a main slip plane; fracture deformations with the granulation and fragmentation processes include the rotation motions;

- Edge dislocations present the asymmetric strains; relation between a density of the edge dislocations and stresses cannot be found in the frame of the classical theory.

Of course the classical elasticity describes almost perfectly the small deformations, but in observations in a near field earthquake zone we meet with a number of the well-recognized insufficiencies, moreover the recoding of the very long seismic waves means that in fact we record the deformation fields, $D_{i k}=\partial u_{k} / \partial x_{i}$, which becomes integrated during the adequate time by a seismometer to reveal in abstraction the long displacement motion $\left(u_{k}=\int \sum\left(\partial u_{k} / \partial x_{i}\right) \Delta x_{i} \mathrm{~d} t ;\right.$ where $\Delta x$ means a rigid element of seismometer platform).

We might mention some numerous attempts used to improve the classical elasticity; however we will confine ourselves to the following:

- The Cosserat brothers' theory of elasticity (Cosserat, E. and F. 1909) included the displacements and rotations;

- The micropolar and micromorphic elastic theories (cf., Eringen and Suhubi, 1964; Mindlin, 1965; Nowacki, 1986; for an advanced review, see Eringen 1999, 2001).

These micropolar and micromorphic theories present a very powerful tool to describe many complicated problems (see, e.g, Teisseyre, 1973; 1974). These theories seem to be too much complicated for an use in common seismological studies and require a knowledge of many additional material constants.

The similar approach can be introduced for the fluids; the molecular strains may present the symmetric and anti-symmetric molecular deformations (Teisseyre, 2009). We will meet there the additional complications when considering the non-laminar and turbulent motions; moreover, the density variation in gases will have a great importance. We will present here the first trials in these directions; however, we should underline that such efforts have been already undertaken in a frame of the micropolar and micromorphic theories (Eringen, 2001).

From the presented reason this contribution is divided into two parts: Fracture earthquake processes and Extreme fluid dynamics;

\section{Fracture earthquake processes}

\subsection{Motion relations for fields released in independent fractures}

Following the Asymmetric Theory (cf., Teisseyre, R. 2009, Teisseyre, 2011) we present the relations for the basic motions and deformations. For the basic fields, displacements, rotations, axial and strains treated as the independent motions we present the related motion equations. For the symmetric strain and for rotation strain we write: 


$$
\begin{gathered}
E_{(i k)}=\frac{1}{2}\left(\frac{\partial u_{k}}{\partial x_{i}}+\frac{\partial u_{i}}{\partial x_{k}}\right) ; \quad \breve{E}_{[i k]}=\frac{1}{2}\left(\frac{\partial \breve{u}_{k}}{\partial x_{i}}-\frac{\partial \breve{u}_{i}}{\partial x_{k}}\right) \\
D_{i k}=\frac{\partial u_{k}}{\partial x_{i}}, \breve{D}_{i k}=\frac{\partial \breve{u}_{k}}{\partial x_{i}} ; E_{i k}=E_{(i k)}+\breve{E}_{[i k]}
\end{gathered}
$$

where their sum present the asymmetric strain field, $E_{i k}$, and the related displacement might be different.

The strains relate to the physical deformations, $D_{i k}=\frac{\partial u_{k}}{\partial x_{i}}$ or $\breve{D}_{i k}=\frac{\partial \breve{u}_{k}}{\partial x_{i}}$; The symmetric strains, $E_{(i k)}$, can be expressed by the derivatives of displacements, $u_{k}$, which may originally relate to the fracture slip motion at seismic source, while the rotation strain represents a rotation on a molecular level, which is given by the expression, $\breve{E}_{[i k]}=\frac{1}{2}\left(\breve{D}_{i k}-\breve{D}_{k i}\right)$, related to the displacements, $\breve{u}_{k}$, (having only the mathematical sense):

$$
\omega_{n}=\frac{1}{2} \varepsilon_{n i k} \omega_{i k}=\frac{1}{2} \varepsilon_{n i k} \frac{\partial \breve{u}_{k}}{\partial x_{i}}, \breve{E}_{[i k]} \equiv \omega_{i k}=\frac{1}{2}\left(\frac{\partial \breve{u}_{k}}{\partial x_{i}}-\frac{\partial \breve{u}_{k}}{\partial x_{i}}\right)
$$

where $\varepsilon_{\text {nik }}=\{1,-1,0\}$ is the fully antisymmetric tensor.

The total asymmetric strains, $E_{i k}$, may be presented as follows:

$$
\begin{gathered}
E_{k l}=E_{(k l)}+\breve{E}_{[k l]}=\delta_{k l} \bar{E}+\hat{E}_{(k l)}+\breve{E}_{[k l]} ; \\
\bar{E}=\frac{1}{3} \sum_{s=1}^{3} E_{(s s)} ; \quad \hat{E}_{(i k)}=E_{(i k)}-\delta_{i k} \frac{1}{3} \sum_{s=1}^{3} E_{(s s)}
\end{gathered}
$$

where the total axial strain, $\bar{E}=-p$, may relate to pressure and the deviatoric strains, $\hat{E}_{(i k)}$, relate to the shear field.

All these strain fields, total axial, deviatoric shear and rotational, can be released at a seismic source quite independently or some of these strains might be mutually related through their relations to the reference displacements, e.g., $\bar{u}_{s}, \hat{u}_{s}, \breve{u}_{s}$ :

$$
\begin{gathered}
\bar{E}=\frac{1}{3} \sum_{s} E_{s s}=\frac{1}{3} \sum_{s} \frac{\partial \bar{u}_{s}}{\partial x_{s}} \text { - total axial strain } \\
\hat{E}_{(i k)}=E_{(i k)}-\delta_{i k} \frac{1}{3} \sum_{s=1}^{3} E_{(s s)}=\frac{1}{2}\left(\frac{\partial \hat{u}_{k}}{\partial x_{i}}+\frac{\partial \hat{u}_{i}}{\partial x_{k}}\right)-\delta_{i k} \frac{1}{3} \sum_{s=1}^{3} \frac{\partial \hat{u}_{s}}{\partial x_{s}} \text { - deviatoric shears } \\
\breve{E}_{[i k]}=\frac{1}{2}\left(\frac{\partial \breve{u}_{k}}{\partial x_{i}}-\frac{\partial \breve{u}_{i}}{\partial x_{k}}\right) \text { - rotation strain }
\end{gathered}
$$

where some inter-relations between the written reference displacements may be related to the join source processes; in general the relation to common, phase shifted, or independent displacements may be expressed as: 


$$
\bar{u}_{s}=\xi^{0} u_{s}, \quad \hat{u}_{s}=e^{0} u_{s}, \breve{u}_{s}=\chi^{0} u_{s} ; \quad\left\{\xi^{0}, e^{0}, \chi^{0}\right\}=\{0, \pm 1, \pm \mathbf{i}\}
$$

Strain rotation has a different sense that a simple rotation motion. However, in relation to the displacement derivatives both might be expressed by the similar mathematical formulae.

We assume that any independent motion or deformation may be represented by the independent equations of motion (cf., Teiseyre and Gorski, 2009, Teisseyre, 2009, Teisseyre, 2011).

The motion equation for deformation, $D_{n i}=\frac{\partial u_{i}}{\partial x_{n}}$, follows from the derivatives of the classic
Newton formula as follows:

$$
\mu \sum_{s} \frac{\partial^{2} u_{i}}{\partial x_{s} \partial x_{s}}-\rho \frac{\partial^{2} u_{i}}{\partial t^{2}}+(\lambda+\mu) \frac{\partial}{\partial x_{i}} \sum_{s} \frac{\partial u_{s}}{\partial x_{s}}=0
$$

Accordingly for symmetric and anti-symmetric strains we obtain:

$$
\begin{gathered}
\mu \sum_{s} \frac{\partial^{2}}{\partial x_{s} \partial x_{s}} E_{(n l)}-\rho \frac{\partial^{2}}{\partial t^{2}} E_{(n l)}=-(\lambda+\mu) \frac{\partial^{2}}{\partial x_{n} \partial x_{l}} \sum_{s} E_{(s s)} ; \quad E_{(n l)}=\frac{1}{2}\left(\frac{\partial u_{l}}{\partial x_{n}}+\frac{\partial u_{n}}{\partial x_{l}}\right) \\
\mu \sum_{s} \frac{\partial^{2}}{\partial x_{s} \partial x_{s}} \breve{E}_{[n l]}-\rho \frac{\partial^{2}}{\partial t^{2}} \breve{E}_{[n l]}=0 ; \quad \breve{E}_{[n l]}=\frac{1}{2}\left(\frac{\partial u_{l}}{\partial x_{n}}-\frac{\partial u_{n}}{\partial x_{l}}\right)
\end{gathered}
$$

where in all these formulae we may use the different reference displacement motions as explained above (see, eqs. 4a-4c); we have also omitted the external forces.

For the constitutional relations, joining stresses and strains, we choice the most simple relations:

$$
\begin{gathered}
S_{(i k)}=2 \mu E_{(i k)}+\lambda \delta_{i k} E_{(s s)} \quad ; \quad S_{[i k]}=2 \mu E_{[i k]} \\
\bar{S}=\left(2 \mu+3 \lambda \delta_{i k}\right) \bar{E} ; \quad \hat{S}_{(i k)}=2 \mu \hat{E}_{(i k)} ; \quad \breve{S}_{[i k]}=2 \mu \breve{E}_{[i k]}
\end{gathered}
$$

and the stresses may be separated for the axial, deviatoric and rotation stresses:

$$
\begin{gathered}
S_{k l}=S_{(k l)}+S_{[k l]}=\delta_{k l} \bar{S}+\hat{S}_{(k l)}+\breve{S}_{[k l]} ; \\
\bar{S}=\frac{1}{3} \sum_{s=1}^{3} S_{(s s)}, \hat{S}_{(i k)}=S_{(i k)}-\delta_{i k} \frac{1}{3} \sum_{s=1}^{3} S_{(s s)}
\end{gathered}
$$

\subsection{Release-rebound fractures and propagation of waves}

We have already mentioned that the fracture source processes could run according to the release - rebound related processes; this can have its influence on a propagation pattern. We follow some result presented by Teisseyre, $(1985,2009,2011)$.

When a total axial stress is constant, the release-rebound system may be described by the linear relations between the time and space derivatives; the unique relations might remind the Maxwell-like ones. To this end we should choice the special coordinate system in which 
the deviatoric strains can be represented by the off- diagonal tensor in the specially chosen coordinate system:

$$
\hat{E}_{(i k)}=E_{(i k)}-\delta_{i k} \frac{1}{3} \sum_{s=1}^{3} E_{(s s)} \rightarrow \hat{E}_{(i k)}=\left[\begin{array}{ccc}
0 & \hat{E}_{(12)} & \hat{E}_{(13)} \\
\hat{E}_{(12)} & 0 & \hat{E}_{(23)} \\
\hat{E}_{(13)} & \hat{E}_{(23)} & 0
\end{array}\right]
$$

In this system we can define the shear vector as $\hat{E}_{i}=\left\{\hat{E}_{(23)}, \hat{E}_{(31)}, \hat{E}_{(12)}\right\}$, and the rotation vector $\breve{E}_{i}$ as $\breve{E}_{i}=\left\{\breve{E}_{[23]}, \breve{E}_{[31]}, \breve{E}_{[12]}\right\}$. We may mention that when using the $4 \mathrm{D}$ approach, the vector $\hat{E}_{i}$ can be defined invariantly (cf., Teisseyre, 2009)

The release-rebound process may mean that a break of molecular bonds on molecular level releases a rotation field, $\partial \breve{E} / \partial t$, and than in a rebound motion will appear, $\operatorname{rot} \hat{E}$, and reversely a release of shear field, $\partial \hat{E} / \partial t$, causes the rebound change: a rotation of the angular strains, rot $\breve{E}$; a shear strain may cause a slip motion at a source.

Such a release-rebound processes are adequately described by the Maxwell-like relations (Teisseyre, 2009, 2011):

$$
\operatorname{rot} \breve{E}-\frac{\partial \hat{E}}{V \partial t}=0, \quad \operatorname{rot} \hat{E}+\frac{\partial \breve{E}}{V \partial t}=0 ; \quad V=V^{S}=\sqrt{\frac{\mu}{\rho}}
$$

From these relations we obtain the wave equations which coincide with the earlier derived formulae (6a) and (6c). When $\sum_{s} \frac{\partial u_{s}}{\partial x_{s}}$ remains constant we obtain the wave relations for rotation and shear fields, which, de facto, are mutually correlated as shown in the previous equation:

$$
\Delta \breve{E}-\frac{\partial^{2} \breve{E}}{V^{2} \partial t^{2}}=0 \text { and } \Delta \hat{E}-\frac{\partial^{2} \hat{E}}{V^{2} \partial t}=0
$$

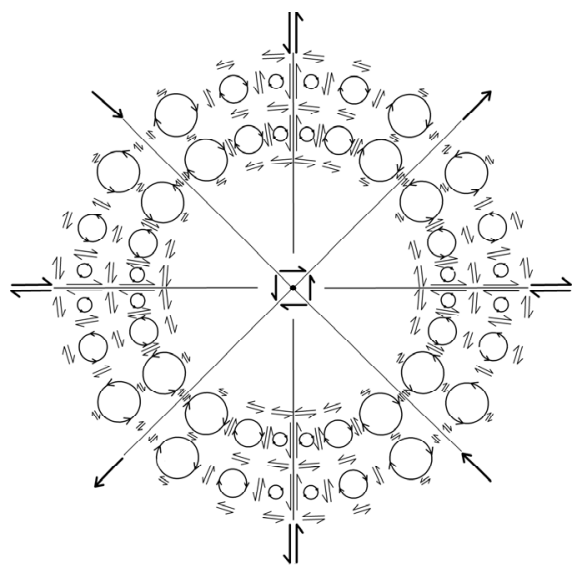

Fig. 1. Wave interaction pattern related to shears and rotation strains. 
The related wave mosaic (Teisseyre and Gorski 2011) explains the interrelated propagation pattern of the shear and rotation motions as presented on Fig. 1.

Such pattern visualizes the wave interactions corresponding to the release-rebound processes at a fracture source.

\subsection{Induced strains}

In a seismic active zones we have a very high concentration of the defects; the classic paper by Eshelby et al. (1951) states that an array of the $n$ linear defects leads to a concentration of the applied external stress field, S, to a multiple value of the applied field at a top of an array:

$$
S^{T o p}=n S
$$

Such stress concentration can form a crack. The Peach - Koehler forces exerted on defect lines (Peach and Koehler, 1950) are related to the dislocation slip vector, b , dislocation line versor, $\mathrm{v}$, and the applied stress field, $S_{s k}$ :

$$
F_{n}=\varepsilon_{n s q} \sum_{k} S_{s k} b_{k} v_{q}
$$

where the fully asymmetric tensor, $\varepsilon_{n s q}$, expresses the vector product between the stress field multiplied by a slip vector and a dislocation line versors.

Two opposite cracks could mutually join to form a bigger crack; in such a process the stresses concentrated at the opposite edges of these cracks (or arrays) become mutually annihilated; that means their energies become released. This simple fracture model (cf., Droste , Teisseyre, 1959) could be applied to a continuum with a high defect content. To this end we should generalize the original Peach - Koehler expression (12) assuming, first, that the stresses, $S_{s k}$, represent an asymmetric field and, second, that we can describe the defect density by means of dislocation slip vector and dislocation line versor:

$$
\alpha_{q k}=v_{q} b_{k}
$$

Then using relations (12) and (13) we can define the induced stresses as product of the Peach-Koehler force and normal to a defect plane, $F_{n} n_{p}$ :

$$
\begin{aligned}
& S_{n p}^{I n d}=S_{(n p)}^{I n d}+S_{[n p]}^{I n d}=F_{n} n_{p}=\varepsilon_{n s q} \sum_{k} \alpha_{q k} S_{s k} n_{p} \\
& S_{(n p)}^{I n d}=\frac{1}{2} \varepsilon_{n s q} \sum_{k} \alpha_{q k} S_{s k} n_{p}+\frac{1}{2} \varepsilon_{p s q} \sum_{k} \alpha_{q k} S_{s k} n_{n} \\
& S_{[n p]}^{I n d}=\frac{1}{2} \varepsilon_{n s q} \sum_{k} \alpha_{q k} S_{s k} n_{p}-\frac{1}{2} \varepsilon_{p s q} \sum_{k} \alpha_{q k} S_{s k} n_{n}
\end{aligned}
$$

The total stresses would means a reorganized stress system due to a defect influence:

$$
S_{n p}^{T}=S_{n p}+S_{n p}^{I n d}=S_{(n p)}+S_{[n p]}+S_{(n p)}^{I n d}+S_{[n p]}^{I n d}
$$


Considering the applied field as given by the constant total axial stresses only (that is applied stresses equivalent to an applied constant pressure, $S_{n p}^{T}=-p \delta_{n p}+S_{n p}^{\text {Ind }}$ ) we obtain from eqs. 14 and 15 that the non diagonal component of asymmetric total stresses will relate to the expressions with a defect contain:

$$
S_{12}^{T}=p\left(\alpha_{23}-\alpha_{32}\right) n_{2}, S_{23}^{T}=p\left(\alpha_{31}-\alpha_{13}\right) n_{3}, S_{31}^{T}=p\left(\alpha_{12}-\alpha_{21}\right) n_{1}
$$

while the axial fields will be given as follows

$$
S_{11}^{T}=\left(\alpha_{23}-\alpha_{32}-1\right) p n_{1}, S_{22}^{T}=\left(\alpha_{31}-\alpha_{13}-1\right) p n_{2}, S_{33}^{T}=p\left(\alpha_{12}-\alpha_{21}-1\right) p n_{3}
$$

Thus, we come to an important expression that a fracture under pressure will run as the shear and strain rotation mutually related processes; this result confirms a known experimental fact that under applied pressure a fragmentation has a shear and rotational character.

\subsection{Seismological networks}

The global seismological network is based only on recording of displacements by the system of seismometers; only in some separate seismically active regions we have the local system of strain-meters and in some places also the devises to record a rotation motion.

However, a desired global system could watch any strain changes and related waves in a global scale.

At a given time moment the recorded displacement data presents a total sum of all deformation released and appears only due to the integration effect of a sum of deformations, $\frac{\partial u_{q}}{\partial x_{p}}$, over an interval, $\Delta x$, which means a length of a seismometer platform much more rigid than soil layers. At a given time moment we obtain:

$$
\Delta u_{k}=\int \frac{\partial u_{k}}{\partial x_{i}} \Delta x_{i}
$$

and the total displacement would be given as:

$$
u_{k}=\int \sum \frac{\partial u_{k}}{\partial x_{i}} \Delta x_{i} \mathrm{~d} t
$$

Only in such a way we might be inform on a displacement amplitude of the long strain waves.

Thus, it is extremely important to have the new word-wide seismic network based on the strain and rotation recording systems. We should note that only in the few seismic active regions such recording systems already exist, this is not enough as the strain waves can propagate over the whole word.

\section{Extreme fluid dynamics}

\subsection{Asymmetric relations for momentum flux}

We follow the asymmetric continuum theory developed in our former papers (Teisseyre, 2007; 2008; 2009; 2011); we assume that beside the symmetric molecular strains (strain rates) 
there appear also the antisymmetric molecular strain rates (spins); for the related molecular stresses we write:

$$
\tilde{S}_{k l}=\tilde{S}_{(k l)}+\tilde{S}_{[k l]}
$$

These fields, stress and strain rates, can be related by means of the following constitutive relations

$$
\begin{aligned}
& \frac{1}{3} \sum_{s} \tilde{S}_{s s}=-p=\frac{1}{3} k \sum_{s} \tilde{E}_{s s} ; \quad \tilde{S}_{k l}=\tilde{S}_{(k l)}+\widetilde{\tilde{S}}_{[k l]}, \quad \tilde{E}_{k l}=\tilde{E}_{(k l)}+\widetilde{\tilde{E}}_{[k l]} \\
& \tilde{S}_{(k l)}^{D}=\eta \tilde{E}_{(k l)}^{D}, \quad \tilde{S}_{[k l]}=\eta \tilde{\tilde{E}}_{[k l]} ; \quad \tilde{S}_{(k l)}^{D}=\tilde{S}_{(k l)}-\frac{1}{3} \delta_{k l} \tilde{S}_{s s}, \quad \tilde{E}_{(k l)}^{D}=\tilde{E}_{(k l)}-\frac{1}{3} \delta_{k l} \tilde{E}_{s s}
\end{aligned}
$$

where $\tilde{S}_{k l}^{D}$ and $\tilde{E}_{k l}^{D}$ mean the deviatoric parts of tensors and $\eta$ is the viscosity; for a simplicity we have assumed the same constant, $\eta$, for symmetric and antisymmetric parts; the points in the considered fluid continuum will have six degrees of freedom: displacement velocity and rotation motion - spin.

The molecular strain rates could be related the derivatives of some reference displacement velocities, $v_{l}$ and $v_{l}^{\prime}$ :

$$
\tilde{E}_{k l}=\frac{1}{2}\left(\frac{\partial v_{l}}{\partial x_{k}}+\frac{\partial v_{k}}{\partial x_{l}}\right), \quad \widetilde{\tilde{E}}_{[k l]}=\frac{1}{2}\left[\frac{\partial v_{l}^{\prime}}{\partial x_{k}}-\frac{\partial v_{k}^{\prime}}{\partial x_{l}}\right]
$$

where the field $v^{\prime}$ may have only a mathematical sense.

For the equal reference velocities, $v^{\prime}=v$, we can obtain the relation between the molecular stresses and displacement velocity as a sum of molecular strain rate and molecular spin (having another sense that a simple point rotation velocity (cf., eqs. 1, 2 and Fig.1):

$$
\tilde{S}_{k l}=\tilde{S}_{(k l)}+\widetilde{\tilde{S}}_{[k l]}=\eta \frac{\partial v_{l}}{\partial x_{k}}=\eta\left(\tilde{E}_{k l}+\widetilde{\tilde{E}}_{[k l]}\right)
$$

A macroscopic transport field, $\mathrm{v}$, we will treat as an imposed external field, while the internal transport related to the rotation velocity could be treated as the additional motion introduced to the system of equations. Thus, in motions with advanced vorticity dynamics we assume that a transport can be related both to displacement velocity and the vortex motions (cf., Teisseyre, 2009).

First we may remember the Navier-Stokes relations:

$$
\frac{\mathrm{d}\left(\rho v_{i}\right)}{\mathrm{d} t}=v_{i} \frac{\partial \rho}{\partial t}+\rho \frac{\partial v_{i}}{\partial t}+\rho v_{s} \frac{\partial v_{i}}{\partial x_{s}}=\eta \frac{\partial^{2} v_{i}}{\partial x_{k} \partial x_{k}}-\tilde{F}_{i}
$$

where $\tilde{\mathrm{F}}$ are the body forces, $\mathrm{v}$ is the displacement velocity, $\eta$ is the dynamic viscosity.

We may present this relation in the different forms, with the independent fields $\tilde{E}_{k i}$ and $\tilde{\tilde{E}}_{[k i]}$, and valid both for $\tilde{E}_{k i}(v)$ and $\tilde{\tilde{E}}_{[k i]}\left(v^{\prime}\right)$ with $v^{\prime}=v$, or with the phase shifted reference displacements, $v^{\prime}= \pm i v$ : 


$$
\frac{\mathrm{d}\left(\rho v_{i}\right)}{\mathrm{d} t} \rightarrow v_{i} \frac{\partial \rho}{\partial t}+\rho \frac{\partial v_{i}}{\partial t}+\rho v_{s} \frac{\partial v_{i}}{\partial x_{s}}=\eta \frac{\partial}{\partial x_{k}}\left(\tilde{E}_{(k i)}+\tilde{\tilde{E}}_{[k i]}\right)-\tilde{F}_{i}
$$

This is an equivalent form expressing a possible relation between the molecular strain and spin fields.

For the molecular symmetric stresses we can write after Landau and Lipshitz (1959; new edition in Russian , 2001, cited as its Polish translantion, 2009):

$$
\begin{gathered}
\tilde{S}_{(i j)}=2 \eta\left(\tilde{E}_{(i j)}-\frac{\delta_{i j}}{3} \sum_{s} \frac{\partial v_{s}}{\partial x_{s}}\right)+\varepsilon \delta_{i j} \sum_{s} \frac{\partial v_{s}}{\partial x_{s}}, \text { or } \\
\tilde{S}_{(i j)}=\eta\left(\frac{\partial v_{j}}{\partial x_{i}}+\frac{\partial v_{i}}{\partial x_{j}}-\frac{2 \delta_{i j}}{3} \sum_{s} \frac{\partial v_{s}}{\partial x_{s}}\right)+\varepsilon \delta_{i j} \sum_{s} \frac{\partial v_{s}}{\partial x_{s}}
\end{gathered}
$$

In our new approach we should add an influence of rotation molecular processes; therefore we introduce the asymmetric fluid viscous stress tensor adding an influence of the antisymmetric molecular stresses (Teisseyre, 2009):

$$
\widetilde{\tilde{S}}_{[i j]}=2 \eta \widetilde{\tilde{E}}_{[i j]}=\eta\left(\frac{\partial v_{j}}{\partial x_{i}}-\frac{\partial v_{i}}{\partial x_{j}}\right)
$$

The total stresses become give as :

$$
\tilde{S}_{i j}=\tilde{S}_{(i j)}+\tilde{S}_{[i j]} ; \quad \tilde{S}_{i j}=2 \eta\left(\frac{\partial v_{j}}{\partial x_{i}}-\frac{\delta_{i j}}{3} \sum_{s} \frac{\partial v_{s}}{\partial x_{s}}\right)+\varepsilon \delta_{i j} \sum_{s} \frac{\partial v_{s}}{\partial x_{s}}
$$

and the total molecular axial strain as:

$$
\sum_{s} \tilde{S}_{s s}=-3 p
$$

Now for the momentum flux we write the modified asymmetric tensor:

$$
\begin{aligned}
T_{k n} & =-S_{k n}+\rho v_{k} v_{n}+\rho \tilde{v}_{k} \tilde{v}_{n} ; \tilde{v}=\{\dot{\tilde{r}}, \tilde{r} \tilde{\omega}, \dot{\tilde{z}}\} \\
T_{(k n)} & =-S_{(k n)}+\rho v_{k} v_{n}+\rho \tilde{v}_{k} \tilde{v}_{n}, T_{[k n]}=-S_{[k n]}
\end{aligned}
$$

or we could write

$$
T_{k n}=-2 \eta\left(\frac{\partial v_{n}}{\partial x_{k}}-\frac{\delta_{k n}}{3} \sum_{s} \frac{\partial v_{s}}{\partial x_{s}}\right)-\varepsilon \delta_{k n} \sum_{s} \frac{\partial v_{s}}{\partial x_{s}}+\rho v_{k} v_{n}+\rho \tilde{v}_{k} \tilde{v}_{n}
$$

We arrive to the general expression for the motion of fluids given as follows

$$
\frac{\partial\left(\rho v_{n}+\rho \tilde{v}_{n}\right)}{\partial t}+\sum_{k} \frac{\partial T_{k n}}{\partial x_{k}}=\rho g \delta_{z n}+F_{n}
$$


and for a mass conservation we will have

$$
\frac{d \rho}{d t}=\frac{\partial \rho}{\partial t}+\sum_{s} \frac{\partial\left(\rho v_{s}\right)}{\partial x_{s}}+\sum_{s} \frac{\partial\left(\rho \tilde{v}_{s}\right)}{\partial x_{s}}=0
$$

\subsection{Vortex transport processes}

In some problems, a macroscopic transport field, $\mathrm{v}$, can be treated as an imposed external field, while the internal transport rotation shall enter into the additional terms introduced to the system of equations. Thus, in motions with advanced vorticity dynamics we assume that a transport can be related both to displacement velocity and the vortex motions; we follow the approach applied to fragmentation and slip in the fracture processes (Teisseyre, 2009).

For great Reynolds number the laminar motions become unstable and the double transport process with displacement velocities and spin motions may generate the micro-vortices; a kind of dynamic vortex structure can be formed, simultaneously undergoing a displacement transport process.

However, under some special conditions, an isolated vortex center can be formed; inside it, the displacement velocity transport might be negligible. However, to consider formally the transport related to such extreme conditions we should return to the basic problem: how to incorporate the spin motion into the system of basic relations.

The independent rotational motions related to angular moment and the variable arm and spin could well describe the vortex transport phenomena (Teisseyre, 2009); we should underline that our approach differs from the classical one defining a vorticity as rotation of transport velocity:

$$
\varsigma=\operatorname{rot} \tilde{v}\left(\text { or } \quad \varsigma_{n}=\varepsilon_{n p i} \partial \tilde{v}_{i} / \partial x_{p}\right)
$$

Following that paper (Teisseyre, 2009) the rotation transport, $\tilde{v}_{k}$, can be given as radial velocity, $\tilde{r}$, angular velocity, $\tilde{\omega}$ and axial velocity, $\tilde{z}$ :

$$
\tilde{v}_{k} \rightarrow\{\tilde{r}, \tilde{\omega}, \tilde{z}\}
$$

We obtain for a rotation transport in the vortices along the z-axis:

$$
\begin{aligned}
& \frac{\mathrm{d}}{\mathrm{d} t}=\frac{\partial}{\partial t}+\tilde{v}_{k} \frac{\partial}{\partial x_{k}} \rightarrow \frac{\mathrm{d}}{\mathrm{d} t}=\frac{\partial}{\partial t}+\tilde{r} \frac{\partial}{\partial r}+\tilde{\omega} \frac{\partial}{\partial \varphi}+\tilde{z} \frac{\partial}{\partial z} \rightarrow \\
& \frac{\mathrm{d}(\rho \tilde{r})}{\mathrm{d} t}=\tilde{r} \frac{\partial \rho}{\partial t}+\rho \frac{\partial \tilde{r}}{\partial t}+\rho \tilde{r} \frac{\partial \tilde{r}}{\partial r}+\rho \tilde{\omega} \frac{\partial \tilde{r}}{\partial \varphi}+\rho \tilde{z} \frac{\partial \tilde{r}}{\partial z} \\
& \frac{\mathrm{d}(\rho \tilde{\omega})}{\mathrm{d} t}=\tilde{\omega} \frac{\partial \rho}{\partial t}+\rho \frac{\partial \tilde{\omega}}{\partial t}++\rho \tilde{r} \frac{\partial \tilde{\omega}}{\partial r}+\rho \tilde{\omega} \frac{\partial \tilde{\omega}}{\partial \varphi}+\rho \tilde{z} \frac{\partial \tilde{\omega}}{\partial z} \\
& \frac{\mathrm{d}(\rho \tilde{z})}{\mathrm{d} t}=\tilde{z} \frac{\partial \rho}{\partial t}+\rho \frac{\partial \tilde{z}}{\partial t}++\rho \tilde{r} \frac{\partial \tilde{z}}{\partial r}+\rho \tilde{\omega} \frac{\partial \tilde{z}}{\partial \varphi}+\rho \tilde{z} \frac{\partial \tilde{z}}{\partial z}
\end{aligned}
$$

At such the vortex motion we obtain from the equations (26-28) for the momentum flux only a simple expression: 


$$
\begin{aligned}
& T_{k n}=\rho \tilde{v}_{k} \tilde{v}_{n} ; \tilde{v}=\{\tilde{r}, \tilde{\omega}, \tilde{z}\}: \\
& \frac{\partial\left(\rho \tilde{v}_{n}\right)}{\partial t}+\sum_{k} \frac{\partial T_{k n}}{\partial x_{k}}=\rho g \delta_{z n}
\end{aligned}
$$

that is

$$
\begin{aligned}
& \frac{\partial(\rho \tilde{r})}{\partial t}+\frac{\partial\left(\rho \tilde{r}^{2}\right)}{\partial r}+\frac{\partial(\rho \tilde{\omega} \tilde{r})}{\partial \varphi}+\frac{\partial(\rho \tilde{r} \tilde{z})}{\partial z}=0 \\
& \frac{\partial(\rho \tilde{\omega})}{\partial t}+\frac{\partial(\rho \tilde{r} \tilde{\omega})}{\partial r}+\frac{\partial\left(\rho \tilde{\omega}^{2}\right)}{\partial \varphi}+\frac{\partial(\rho \tilde{\omega} \tilde{z})}{\partial z}=0 \\
& \frac{\partial(\rho \tilde{z})}{\partial t}+\frac{\partial(\rho \tilde{r} \tilde{z})}{\partial r}+\frac{\partial(\rho \tilde{\omega} \tilde{z})}{\partial \varphi}+\frac{\partial\left(\rho \tilde{z}^{2}\right)}{\partial z}=\rho g \delta_{z n}
\end{aligned}
$$

and for a mass conservation we will have

$$
\frac{d \rho}{d t}=\frac{\partial \rho}{\partial t}+\tilde{r} \frac{\partial \rho}{\partial r}+\tilde{\omega} \frac{\partial \rho}{\partial \varphi}+\tilde{z} \frac{\partial \rho}{\partial z}=0
$$

These relation present the dynamic relation under a given thermodynamical condition.

At constant thermodynamical condition, $\frac{\partial \rho}{\partial t}=0$, we obtain:

$$
\begin{aligned}
& \rho \frac{\partial \tilde{r}}{\partial t}+\frac{\partial\left(\rho \tilde{r}^{2}\right)}{\partial r}+\frac{\partial(\rho \tilde{\omega} \tilde{r})}{\partial \varphi}+\frac{\partial(\rho \tilde{r} \tilde{z})}{\partial z}=0 \\
& \rho \frac{\partial \tilde{\omega}}{\partial t}+\frac{\partial(\rho \tilde{r} \tilde{\omega})}{\partial r}+\frac{\partial\left(\rho \tilde{\omega}^{2}\right)}{\partial \varphi}+\frac{\partial(\rho \tilde{\omega} \tilde{z})}{\partial z}=0 \\
& \rho \frac{\partial \tilde{z}}{\partial t}+\frac{\partial(\rho \tilde{r} \tilde{z})}{\partial r}+\frac{\partial(\rho \tilde{\omega} \tilde{z})}{\partial \varphi}+\frac{\partial\left(\rho \tilde{z}^{2}\right)}{\partial z}=\rho g \delta_{z n}
\end{aligned}
$$

and for a mass conservation we will have

$$
\frac{d \rho}{d t}=\tilde{r} \frac{\partial \rho}{\partial r}+\tilde{\omega} \frac{\partial \rho}{\partial \varphi}+\tilde{z} \frac{\partial \rho}{\partial z}=0
$$

A complex system presents relations given by the equations 25 and 33 . We might solve these equations for two cases; first with constant density $\rho=\rho_{0}$ outside the vortices, (e.g., for liquids), given by the relations (25) and with a variable density inside the vortices, $\rho(r, \varphi, z)$; still this complex system with relations (25 and 33) will remain quite complicated. Outsite the vortices we could have the curvilinear system of flow around and between the vortices.

\subsection{Stationary vortex relations}

We will confine our consideration to a single vortex space, the related stationary equations at constant thermodynamical condition with $\frac{\partial \rho}{\partial t}=0$ become: 


$$
\begin{aligned}
& \frac{\partial\left(\rho \tilde{r}^{2}\right)}{\partial r}+\frac{\partial(\rho \tilde{\omega} \tilde{r})}{\partial \varphi}+\frac{\partial(\rho \tilde{r} \tilde{z})}{\partial z}=0 \\
& \frac{\partial(\rho \tilde{r} \tilde{\omega})}{\partial r}+\frac{\partial\left(\rho \tilde{\omega}^{2}\right)}{\partial \varphi}+\frac{\partial(\rho \tilde{\omega} \tilde{z})}{\partial z}=0 \\
& \frac{\partial(\rho \tilde{r} \tilde{z})}{\partial r}+\frac{\partial(\rho \tilde{\omega} \tilde{z})}{\partial \varphi}+\frac{\partial\left(\rho \tilde{z}^{2}\right)}{\partial z}=\rho g \delta_{z n}
\end{aligned}
$$

and

$$
\tilde{r} \frac{\partial \rho}{\partial r}+\tilde{\omega} \frac{\partial \rho}{\partial \varphi}+\tilde{z} \frac{\partial \rho}{\partial z}=0
$$

Note that such the flows can occur effectively for the Reynolds numbers above the critical value. It would be possible to solve these stationary cases with a help of the following types of relations:

$$
\begin{aligned}
& \tilde{r}=\tilde{r}_{0}\left[1-\varepsilon \frac{\varphi}{2 \pi}\right] \exp (-\alpha z) \exp (\gamma r), \tilde{\omega}=\tilde{\omega}_{0}\left[1-\varsigma \frac{\varphi}{2 \pi}\right] \exp (\beta z) \exp (\delta r) \\
& \tilde{z}=\tilde{z}_{0} \exp (\gamma z) \exp (\kappa r)
\end{aligned}
$$

where $\alpha>0, \beta>0$.

The profiles of related vortices, $\tilde{r}(z)$, may differ from almost linear for very small, $\alpha$ ( that is for $\exp [-\alpha z] \approx 1-\alpha z$ ) up to very rapidly decreasing, $\tilde{r}_{0}(z)$, for the great $\alpha$ values. The spin motion should adequately increase and vertical vortex component, $\tilde{z}$, might increase or decrease.

We may simplify these relations assuming that density can change only along the $z$ - axis:

$$
\rho=\rho_{0} \exp [\vartheta z]
$$

and further more we might put instead of eq. 35:

$$
\tilde{r}=\tilde{r}_{0} \exp (-\alpha z) \exp (\chi r), \quad \tilde{\omega}=\tilde{\omega}_{0} \exp (\beta z) \exp (\delta r), \quad \tilde{z}=\tilde{z}_{0} \exp (\gamma z) \exp (\kappa r)
$$

Here to solve the equations

$$
\begin{aligned}
& \rho \frac{\partial\left(\tilde{r}^{2}\right)}{\partial r}+\rho \frac{\partial(\tilde{r} \tilde{z})}{\partial z}+\tilde{r} \tilde{z} \frac{\partial \rho}{\partial z}=0 \\
& \rho \frac{\partial(\tilde{r} \tilde{\omega})}{\partial r}+\rho \frac{\partial(\tilde{\omega} \tilde{z})}{\partial z}+\tilde{\omega} \tilde{z} \frac{\partial \rho}{\partial z}=0 \\
& \rho \frac{\partial(\tilde{r} \tilde{z})}{\partial r}+\rho \frac{\partial\left(\tilde{z}^{2}\right)}{\partial z}+\tilde{z}^{2} \frac{\partial \rho}{\partial z}=\rho g \delta_{z n}
\end{aligned}
$$

and 


$$
\tilde{r} \frac{\partial \rho}{\partial r}+\tilde{\omega} \frac{\partial \rho}{\partial \varphi}+\tilde{z} \frac{\partial \rho}{\partial z}=0
$$

we will have enough parameters, $\vartheta, \alpha, \beta, \gamma, \chi, \delta, \kappa$, to solve the same number of linear equations as the three equations (34a) will split into six relations: three additional equtions should express the equality of the exponnetial parameters appearing there.

Note that such the flows can occur effectively for the Reynolds numbers above the critical value.

\section{Conclusions}

Our consideration on the continuum theories used in seismology clearly indicate that the global seismic recording network based on the 3-components seismometer stations is quite insufficient to trace the strain waves, especially the long period waves spreading inside a whole Earth interior. To confirm this important need of a global strain and rotation seismic network we should mention that in some seismically very active regions the strain-meter system and rotation sensor system already exist.

Following this new approach to solid state continuum theory, we presented a new asymmetric molecular theory for fluids with a hope that this new approach opens a way to consider a mechanical model of vortex and turbulence motions; here, we have confined our considerations to processes under a constant thermodynamical condition.

\section{References}

Cosserat, E., and F. Cosserat (1909), Théorie des Corps Déformables, A. Hermann et Fils, Paris.

Droste, Z., and R. Teisseyre (1959), The mechanism of earthquakes according to dislocation theory, Sci. Rep. Tohoku Univ., Ser. 5, Geophys. 11, 1, 55-71.

Eringen, A.C. (1999), Microcontinuum Field Theories, Springer Verlag, Berlin.

Eshelby, J.D., F.C. Frank, and F.R.N. Nabarro (1951), The equilibrium of linear arrays of dislocations, Philos. Mag. 42, 351-364.

Eringen, A.C. (2001), Microcontinuum Field Theories, Springer Verlag, Berlin.

Eshelby, J.D., F.C. Frank, and F.R.N. Nabarro (1951), The equilibrium of linear arrays of dislocations, Philos. Mag. 42, 351-364.

Landau, L.D. and Lifshitz, J.M.. 1959, Fluid mechanics (Theoretical Physics, v. 6), (translated from Russian by J.B. Sykes and W.H. Reid), London, Pergamon Press, pp 536.

Landau, L.D. and Lifszyc, J.M.. 2009, Hydrodynamika, (in Polish) Wydawnictwo Naukowe PWN Warszawa, pp 671.

Lee W.H.K., Celebi, M., Todorovska M.I. and Igel, H., 2009, Introduction to Special issue on Rotational Seismology and Engineering Applications, Bull. Seismol. Soc. Am. 62, no. 2B 945-957.

Mindlin R.D,1965, On the equations of elastic materials with microstructure, Int. J. Solid Struct., vol 1(1), p. 73

Nowacki, W. 1986, Theory of Asymmetric Elasticity, PWN, Warszawa and Pergamon Press.

Peach, M., and J.S. Koehler (1950), The forces exerted on dislocations and the stress fields produced by them, Phys. Rev. 80, 436-439. 
Teisseyre, R. (1973), Earthquake processes in a micromorphic continuum, Pure Appl. Geophys. 102, 15-28.

Teisseyre, R. (1974), Symmetric micromorphic continuum: wave propagation, point source solutions and some applications to earthquake processes. In: P. Thoft-Christensen (ed.), Continuum Mechanics Aspects of Geodynamics and Rock Fracture Mechanics, 201244.

Teisseyre, R. (1985), New earthquake rebound theory, Phys. Earth Planet. Inter. 39, 1, 1-4.

Teisseyre, R. (2009), Tutorial on new development in physics of rotation motions, Bull. Seismol. Soc. Am. 99, 2B, 1028-1039.

Teisseyre R, 2010, Fluid Theory with Asymmetric Molecular Stresses: Difference between Vorticity and Spin Equations, Acta Geophys. vol 58, 6, 1056-1071T

Teisseyre, R. (2011), Why rotation seismology: Confrontation between classic and asymmetric theories, Bull. Seismol. Soc. Am. 101, 4 , 1683-1691.

Teisseyre, R., and M. Górski ( 2009), Fundamental deformations in asymmetric continuum: Motions and fracturing, Bull. Seismol. Soc. Am. 99, 2B, 1028-1039. 


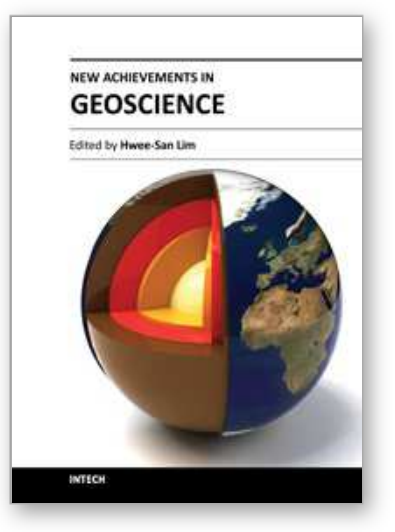

\author{
New Achievements in Geoscience \\ Edited by Dr. Hwee-San Lim
}

ISBN 978-953-51-0263-2

Hard cover, 212 pages

Publisher InTech

Published online 23, March, 2012

Published in print edition March, 2012

New Achievements in Geoscience is a comprehensive, up-to-date resource for academic researchers in geophysics, environmental science, earth science, natural resource managements and their related support fields. This book attempts to highlight issues dealing with geophysical and earth sciences. It describes the research carried out by world-class scientists in the fields of geoscience. The content of the book includes selected chapters covering seismic interpretation, potential field data interpretation and also several chapters on earth science.

\title{
How to reference
}

In order to correctly reference this scholarly work, feel free to copy and paste the following:

Teisseyre Roman (2012). Asymmetric Continuum Theories - Fracture Processes in Seismology and Extreme Fluid Dynamics, New Achievements in Geoscience, Dr. Hwee-San Lim (Ed.), ISBN: 978-953-51-0263-2, InTech, Available from: http://www.intechopen.com/books/new-achievements-in-geoscience/asymmetriccontinuum-theories-fracture-processes-in-seismology-and-extreme-fluid-dynamics

\section{INTECH}

open science | open minds

\section{InTech Europe}

University Campus STeP Ri

Slavka Krautzeka 83/A

51000 Rijeka, Croatia

Phone: +385 (51) 770447

Fax: +385 (51) 686166

www.intechopen.com

\section{InTech China}

Unit 405, Office Block, Hotel Equatorial Shanghai

No.65, Yan An Road (West), Shanghai, 200040, China

中国上海市延安西路65号上海国际贵都大饭店办公楼 405 单元

Phone: +86-21-62489820

Fax: +86-21-62489821 
(C) 2012 The Author(s). Licensee IntechOpen. This is an open access article distributed under the terms of the Creative Commons Attribution 3.0 License, which permits unrestricted use, distribution, and reproduction in any medium, provided the original work is properly cited. 